\title{
EFFECT OF PIOGLITAZONE ON HEPATIC ULTRA- STRUCTURE AND METABOLIC CHANGES INDUCED BY A HIGH SUCROSE DIET IN RATS
}

\author{
${ }^{1}$ Mohamed D. Morsy, ${ }^{2}$ Ahmed S. Khashaba, ${ }^{3}$ Ahmed I. Agamy and ${ }^{4}$ Refaat A. Eid \\ ${ }^{1}$ Department of Physiology, College of Medicine, King Khalid University, Saudi Arabia and Menoufiya University, Egypt \\ ${ }^{2}$ Physiology Medicine Riyadh Colleges of Dentistry and Pharmacy, Saudi Arabia \\ ${ }^{3}$ Department of Physiology, College of Medicine, Benha University, Egypt \\ ${ }^{4}$ Department of Pathology, College of Medicine, King Khalid University, Saudi Arabia
}

Received 2014-02-15; Revised 2014-02-21; Accepted 2014-04-23

\begin{abstract}
Pioglitazone (Pio) is a PPAR- $\gamma$ agonist insulin sensitizer has anti-inflammatory activity. Our novel aspect was to investigate its hepatic anti-inflammatory activity at the level of ultra-structure and enzymatic changes in a high sucrose diet animal model. Forty male Sprague Dawley rats were divided into four equal groups: Control; control Pio; high sucrose diet; high sucrose diet Pio treated groups. Fourteen weeks later, serum glucose, insulin, lipogram, gamma glutamyle transferase, alanine transaminase, aspartate transaminase, alkaline phosphatase, fetuin-A and adiponectin levels were measured. Hepatic tissue homogenate levels of tumor necrosis factor- $\alpha$, interleukin- 6 and myeloperoxidase activity were determined. Microscopic and ultra-structure hepatic changes were conducted in all animal groups. Administration of Pio in HS+Pio group reduced significantly the hepatic inflammatory markers and the hepatic enzymes compared with HS group. Both light and electron microscopic examination revealed a great improvement of the hepatic tissue with Pio treatment. This study suggested that Pio treatment in obesity; in addition to insulin sensitizing activity; could protect the liver from the development of hepatic inflammation induced by a high sucrose diet not only at the enzymatic but also at ultra-structure levels.
\end{abstract}

Keywords: Chronic Hepatic Inflammation, High Sucrose Diet, Pioglitazone, Hepatic Ultra-Structure Changes

\section{INTRODUCTION}

High caloric intake has been increasing steadily in different societies carrying the risk for the development of obesity, diabetes, hepatic and cardiovascular complications (Nielsen and Popkin, 2004). Sugarsweetened beverages contain up to $15 \%$ sucrose promotes obesity and Insulin Resistance (IR) development (Collino et al., 2010). Although the mechanism of IR development not yet exactly known, it has been established that chronic inflammation in key metabolic tissues, including the liver due to fat accumulation is an important pathogenic factor in IR development (Bashir et al., 2014). Hepatic and systemic inflammatory cytokines produced in obesity activate intracellular kinases which capable to inhibit key elements of insulin signaling pathway with subsequent development of insulin resistance (Al-Musa and AL-Hashem, 2014). Tumor Necrosis Factor- $\alpha$ (TNF- $\alpha$ ) and Interleukin-6 (IL-6) among several hepatic cytokines are playing an essential role in hepatic inflammatory conditions. Also, Fetuin-A is a recent discovered hepatokines produced exclusively by the liver in chronic inflammatory conditions. It has been reported that fetuin-A binds with the insulin receptor tyrosine kinase causing its inhibition in the hepatocytes with subsequent suppression of the insulin signal transduction resulting in insulin resistance (Rauth et al., 1992). A reduction in the serum Saudi Arabia and Menoufiya University, Egypt Tel: +966544495223 Fax: +96672418194 
adiponectin level in obesity decreases the insulin sensitivity which leads to aggravation of the adverse effects of inflammatory mediators (Ismail et al., 2014). Adiponectin receptors type 2 are primarily expressed in hepatocytes (Lee et al., 2008) and their down expression in obesity induce liver enzymes over activity involved in the gluconeogenesis and the inflammatory hepatic processes result in inhibition of insulin sensitivity and decreases glucose uptake leads to more severe hepatic inflammatory reaction (Kadowaki et al., 2008).

Several trials have been preformed to alleviate the insulin resistance and the inflammatory hepatic reaction developed in obesity. Peroxisome Proliferator-Activated Receptors (PPARs) are ligand-dependent transcription factors regulate target gene expression by binding to a specific peroxisome proliferator response elements in enhancer sites of regulated genes (Michalik et al., 2006). Moller and Berger (2003) reported that, PPAR- $\gamma$ agonists beyond their insulin-sensitizing efficacy; has antiinflammatory properties. Pioglitazone is a PPAR- $\gamma$ agonist belongs to the Thiazolidinedione (TZD) class of antidiabetic insulin sensitizers. It is able to manage obesity induced insulin resistance (Gerstein et al., 2006). However, to date, few studies carried out on the anti-inflammatory hepatic activity of pioglitazone in addition to its insulin sensitizing effect in obesity and metabolic syndrome.

High sucrose diet induces hepatic fat accumulation, with subsequent insulin resistance and chronic hepatic inflammation leading to overproduction of hepatic inflammatory markers. In addition, the reduction of adiponectin level in obesity aggravates hepatic metabolic disturbances and inflammation. A vicious circle could develop between the IR and the chronic hepatic inflammatory process leading to more and more hepatic inflammatory reaction and insulin resistance. Pioglitazone as PPAR- $\gamma$ agonist has anti-inflammatory activity in addition to its insulin sensitizing effect reduces the hepatic inflammatory markers and increases adiponectin level. From the previous data, we could expect the potential protective effect of PPAR- $\gamma$ agonist pioglitazone on the developing hepatic inflammatory process associated with high caloric intake by high sucrose diet.

The present study was undertaken to determine the effect of the long term administration of the selective PPAR- $\gamma$ agonist pioglitazone on hepatic inflammatory reaction induced by a high sucrose diet in rats. We also try to uncover the different anti-inflammatory mechanisms of Pio and its relation to adiponectin and fetuin-A levels in the hepatic inflammatory cascade in such animal model. The novel point in our research is to investigate the hepatic ultra-structure changes occur in the experimental and in the Pio treated animals.

\section{MATERIALS AND METHODS}

\subsection{Experimental Animals}

All animals received human care in compliance with the Public Health Service Policy on Human Care and Use of Laboratory Animals, published by the National Institutes of Health and were approved by the Ethical Committee of College of Medicine, King Khalid University, Saudi Arabia. This study followed a randomized-controlled animal experiment design. Forty male Sprague-Dawley rats were obtained from animal house of the physiology department, College of Medicine, King Khalid University, Saudi Arabia, weighed between 150-200 gm. Animals were fed with a standard chow diet, water, ad libitum and they were housed in the animal house of College of Medicine with a 12:12-h light/dark cycle. The rats were randomly divided into four groups $(\mathrm{n}=10)$ as follows: Control rats (C) which were given $1 \mathrm{ml}$ distilled water daily through oral gavage; Control Pio treated rats $(\mathrm{C}+\mathrm{Pio})$; which were the animals treated with Pio (Sigma-Aldrich) in a daily dose of $10 \mathrm{mg} \mathrm{kg}^{-1}$ dissolved in one $\mathrm{ml}$ volume of $0.5 \%$ sodium carboxy-methylcellulose by oral gavage (Koufany et al., 2008); A high sucrose diet group (HS) which were given daily $3 \mathrm{~mL}$ of a thirty- percent sucrose solution by oral gavage for 14 weeks (Lombardo et al., 1996; Muñoz Cano et al., 2013). A high sucrose diet pioglitazone treated group (HS+Pio) which were given a high sucrose solution as HS group and were treated with Pio as $\mathrm{C}+\mathrm{Pio}$ group. The body weight for each rat was determined at the start and at the end of the experimental protocol (14 weeks). The percent changes of the body weight were determined.

\subsection{Blood Sampling and Biochemical Measurements}

At the end of the experimental protocol and after overnight fasting, retro-orbital blood samples were obtained through non-heparinized capillary tubes. The samples were allowed to clot for $20 \mathrm{~min}$ in a $37^{\circ} \mathrm{C}$ water bath centrifuged at $14,000 \mathrm{rpm}$ for $10 \mathrm{~min}$ for serum separation and were used for different determination.

\subsection{Determination of Serum Glucose, Total Cholesterol, Triglyceride, HDL-C and LDL-C}

The serum glucose was determined using the glucose oxidase method. The lipid profile estimation was performed 
using commercially available kits for the total cholesterol, triglycerides and HDL-C (BioMerieux, France) whereas the LDL-C was mathematically calculated.

\subsection{Determination of Serum Albumin, Gamma- Glutamyle Transferase (GGT), Alanine Transaminase

$\begin{array}{lr}\text { Aminotransferase } & \text { (AST), } \\ \text { Phosphatase (ALP) } & \end{array}$

The serum albumin, GGT, ALT, AST and ALP were determined by an enzymatic colorimetric method using Randox reagent kits (Sigma-Aldrich).

\subsection{Determination of Fasting Serum Insulin Level}

Determination of serum insulin was performed using an ELISA kit at wavelength 450 NM. (BioSource Europe, Nivelles, Belgium) according to the manufacturer's instruction (Matthews et al., 1985).

\subsection{Calculation of HOMA Index}

The euglycemic hyperinsulinemic clamp is the standard method for measuring insulin resistance. We used a common method using the homeostasis model for HOMA of IR follows the equation: HOMA = fasting glucose (mmol $\left.\mathrm{L}^{-1}\right) \times$ fasting insulin $\left(\mathrm{mU} \quad \mathrm{L}^{-1}\right) / 22.5$. Typically, a HOMA value $>2$ is used to identify significant IR (Matthews et al., 1985).

\subsection{Determination of Serum Adiponectin Level}

Serum adiponectin determination was performed using the rat adiponectin ELISA kit (B-Bridge international, Inc.), according to the manufacturer's instructions (Kubota et al., 2002).

\subsection{Determination of Serum Fetuin-A Level}

Determination of the serum fetuin-A (AssayMax Human alpha-2-HS-Glycoprotein-AHSG-ELISA Kit; Assay pro, St. Charles, MO, USA) was assayed using ELISA kits according to the manufacturer's instructions (Gunduz et al., 2011).

\subsection{Preparation of Hepatic Tissue Homogenate and Histopathological Hepatic Preparation}

After obtaining blood samples, all rats were sacrificed using the lethal dose of thiopental sodium by IP injection. The rat's abdomens were opened and the rat's whole livers were dissected, excided and weighed individually. Then, part of the rat's right liver lobes for each animal was cut and fixed in $10 \%$ formalin then was embedded in paraffin blocks and stained with hematoxylin and eosin for light microscopic examination. The other parts of the right liver lobes were cut into $2-3 \mathrm{~mm}^{3}$ and immediately fixed in $2.5 \%$ glutaraldehyde in $0.1 \mathrm{M}$ sodium cacodylate buffer, $\mathrm{pH} 7.2$ at $4 \mathrm{o} \mathrm{C}$ for $2-3 \mathrm{~h}$. Samples were post-fixed in $1 \%$ osmium tetroxide, dehydrated in an ascending series of ethyl alcohol and embedded in Spurr's resin. Semi-thin sections $(0.5 \mu \mathrm{m})$ were stained with toluidine blue. Ultrathin sections were stained with uranyl acetate and lead citrate and examined in a Jeol JEM-1011 Transmission Electron Microscope (TEM) at $80 \mathrm{KV}$ to observe the hepatic ultra-structure changes by electron microscope. Electron microscope was used to assess the degree of hepatic damage. While the rat's left liver lobes for each rat was homogenized using an Omni tissue homogenizer (Omni international, Gainesville, VA, USA) in ice-cold buffer [0.1 M phosphate, $\mathrm{pH} 7.4,1 \mathrm{mM}$ EDTA, $10 \mu \mathrm{M}$ indomethacin (Cayma Chemical, Ann Arbor, MI, USA)] using a tube pestle. Acetone was added ( $2 \times$ sample volume) and samples were centrifuged at $1500 \times \mathrm{g}$ for $10 \mathrm{~min}$ at $4^{\circ} \mathrm{C}$. The supernatants were then stored at $-80^{\circ} \mathrm{C}$ for determination of hepatic tissue homogenate levels of TNF- $\alpha$ and IL-6 and Myeloperoxidase activity (MPO). At the end of the experiment, the visceral, epididymal and retroperitoneal fats were collected and weighed for each rat individually to ensure the occurrence of general obesity.

\subsection{Liver Homogenate TNF- $\alpha$ and IL-6 assay}

Hepatic TNF- $\alpha$ and IL-6 levels were determined with a double antibody sandwich ABC-ELISA using a rat TNF- $\alpha$ and IL- 6 kits according to the manufacturer's instructions (Shanghai Senxiong Technology industry Co. Ltd, China). The samples were compared with the standard curve and expressed as pg/mL (Xu et al., 2008).

\subsection{Liver Homogenate MPO Activity Assay}

The myeloperoxidase activity in hepatic tissue was determined by the spectrophotometric method. This method uses 3, 3', 5, 5'-Tetramethyl Benzidine (TMB) as an oxidizable dye and the reaction was started by adding Hydrogen Peroxide $\left(\mathrm{H}_{2} \mathrm{O}_{2}\right)$ in the medium (Xu et al., 2008). The assay kits were purchased from Nanjing Jiancheng Bioengineering Institute (Nanjing, China).

\subsection{Statistical Analysis}

The data were expressed as mean \pm Standard Deviation (SD). The data were processed and analyzed using the SPSS version 10.0 (SPSS, Inc., Chicago, Ill., USA). One-way ANOVA was done followed by Tukey's post hoc test. Pearson correlation statistical analysis was done for detection of a 
probable significance between two parameters. The results were considered significant if $\mathrm{p} \leq 0.05$.

\section{RESULTS}

Pioglitazone treatment in normal rats in the $\mathrm{C}+\mathrm{Pio}$ group did not produce any significant changes in all measured parameters and the light and the electron microscopic histopathological examination compared with the $\mathrm{C}$ group except the body weight which increased significantly by Pio treatment compared with the control group (Table 1and 2).

\subsection{Body Weight, Liver Weight, Liver/Body Weight Ratio, Visceral, Epididymal And Retroperitoneal Fats}

High sucrose diet in rats resulted in significant increased in the total body weight, liver weight, the liver/total body weight ratio, visceral, epididymal and retroperitoneal fat $(367 \pm 32,18.25 \pm 1.7,5.97 \pm 0.544$, $7.9 \pm 0.75,17.1 \pm 1.22,16.3 \pm 1.53$ respectively) compared with the control group $(168 \pm 17,5.85 \pm 0.30,3.11 \pm 0.151$, $3.6 \pm 0.31,7.1 \pm 0.55,7.5 \pm 0.69$ respectively). Pio treatment decreased significantly all these parameters $(7.21 \pm 1.1$, $2.92 \pm 0.264,4.1 \pm 0.39,10.2 \pm 0.97,8.3 \pm 0.71$ respectively) except the total body weight which increased significantly $(375 \pm 24)$ compared with the high sucrose diet group (Table 1).

\subsection{Serum Glucose, Insulin and HOMA IR Index}

The present study showed that, high sucrose diet rats resulted in significant elevation of the serum levels of glucose, insulin and HOMA-IR index (136.2 \pm 12.3 , $7.61 \pm 1.02,3.314 \pm 0.027$ respectively) compared with the control group $(71.3 \pm 6.3, \quad 3.83 \pm 0.36, \quad 0.821 \pm 0.015$ respectively). Oral Pio treatment in $\mathrm{HS}+\mathrm{Pio}$ produced a significant reduction in the serum levels of glucose, insulin and HOMA-IR index (95.1 $\pm 8.6,4.97 \pm 0.74$, $1.053 \pm 0.014$ respectively) compared with the HS group (Table 2 and Fig. 3).

\subsection{Serum Cholesterol, Triglyceride, LDL and HDL Levels}

The high sucrose group showed a significant elevation of the serum levels of cholesterol, triglyceride, LDL (132.5 $\pm 13.4,129.6 \pm 12.8,64.8 \pm 6.5$ respectively) whereas there was a significant reduction in the serum level of HDL $(28.5 \pm 2.7)$ compared with the control group (84.6 $\pm 7.5, \quad 91.3 \pm 8.2, \quad 24.7 \pm 3.4$ respectively), $(46.8 \pm 5.1)$. Treatment with Pio in HS+Pio rats resulted in a significant reduction in the cholesterol, triglyceride and LDL levels $(93.8 \pm 7.9,106.4 \pm 9.5,36.80 \pm 3.4)$ with a significant elevation of the serum level of HDL (35.2 \pm 2.6$)$ compared with the HS group (Table 2).

\subsection{Serum Fetuin-A and Adiponectin levels}

The serum fetuin-A level increased significantly whereas the serum adiponectin level decreased significantly in the HS group $(251.2 \pm 23.43,14.51 \pm 1.33$ respectively) compared with the control group $(132.9 \pm 12.08,23.92 \pm 2.18$ respectively). Pio treatment in the HS+Pio group produced a significant reduction in the serum fetuin-A $(172.8 \pm 16.94)$ with a significant elevation of serum adiponectin $(22.16 \pm 2.15)$ levels compared with the HS group (Fig. 2a and b).

\subsection{Serum GGT, ALT, AST, ALP and Albumin}

High sucrose diet in rats resulted in a significant elevation of hepatic serum enzymes GGT, ALT, AST and ALP levels $(1.72 \pm 0.16,141.3 \pm 13.41,265.4 \pm 25.37$, $223.7 \pm 21.67$ respectively) with a significant reduction in the serum albumin level $(21.49 \pm 3.35)$ compared with the control group $(0.76 \pm 0.08,44.3 \pm 4.56,113.7 \pm 5.62$, $116.2 \pm 10.22$ respectively), (41.12 \pm 4.14$)$. Pioglitazone treatment significantly decreased the serum levels of GGT, ALT, AST and ALP $(0.89 \pm 0.07,65.6 \pm 6.23$, $137.6 \pm 11.92, \quad 134.2 \pm 12.62$ respectively) with a significant increase in the serum albumin $(32.24 \pm 2.94)$ compared with the HS group (Fig. 1 and 3c).

\subsection{Correlation of The Serum Fetuin-A With: Serum Adiponectin And Serum GGT}

There is a significant negative correlation between serum fetuin-A and adiponectin levels $(r=-0.8836)$ $(\mathrm{p}<0.0001)$ for the all groups (Fig. 2a). There is a significant positive correlation between the serum fetuinA and the serum GGT levels $(r=0.7062)(p<0.0001)$ for the all groups (Fig. 4).

\subsection{Hepatic Tissue Homogenate Levels of TNF- $\alpha$, IL-6 and MPO Activity}

High sucrose diet in rats resulted in a significant elevation of hepatic tissue inflammatory markers TNF- $\alpha$ and IL-6 levels and MPO activity (4.38 \pm 0.41 , $25.24 \pm 2.57,0.43 \pm 0.06$ respectively) compared with the control group $(1.94 \pm 0.18, \quad 12.72 \pm 1.26, \quad 0.23 \pm 0.04$ respectively). Treatment with PPAR $-\gamma$ agonist Pio produced a significant reduction in hepatic homogenate levels of TNF- $\alpha$ and IL- 6 levels and MPO activity $(3.15 \pm 0.31, \quad 19.21 \pm 1.53, \quad 0.32 \pm 0.05 \quad$ respectively) compared with the HS group (Fig. 2b, c and d). 
Mohamed D. Morsy et al. / American Journal of Applied Sciences 11 (7): 1087-1098, 2014

Table 1. Effect of pioglitazone treatment for 14 weeks in a rat model of high sucrose diet on: Body weight; Liver weight; liver weight/Total body weight; Visceral fat; Epididymal and Retroperitoneal fats

\begin{tabular}{lllll}
\hline & C & C+Pio & HS & HS+Pio \\
\hline Body weight (g) & $168 \pm 17$ & $250 \pm 19^{\ddagger}$ & $367 \pm 32^{\ddagger}$ & $375 \pm 24^{\ddagger}$ \\
Liver weight (g) & $5.85 \pm 0.3$ & $5.91 \pm 0.8$ & $18.25 \pm 1.7^{\ddagger}$ & $7.21 \pm 1.1^{\ddagger \S}$ \\
Relative liver weight/Total body weight (\%) & $3.11 \pm 0.151$ & $2.81 \pm 0.125$ & $5.97 \pm 0.544^{\ddagger}$ & $2.92 \pm 0.264^{\S}$ \\
Visceral fat (g) & $3.6 \pm 0.31$ & $3.3 \pm 0.41$ & $7.9 \pm 0.75^{\ddagger}$ & $4.1 \pm 0.39^{\S}$ \\
Epididymal fat (g) & $7.1 \pm 0.55$ & $7.3 \pm 0.62$ & $17.1 \pm 1.22^{\ddagger}$ & $10.2 \pm 0.97^{\ddagger \S}$ \\
Retroperitoneal fat (g) & $7.5 \pm 0.69$ & $8.1 \pm .82$ & $16.3 \pm 1.53^{\ddagger}$ & $8.3 \pm 0.71^{\S}$
\end{tabular}

C: Control group; C+Pio: Control Pio treated group; HS: High sucrose diet group; HS+Pio: High sucrose diet pioglitazone treated group. Results are expressed as means \pm SD $(n=10)$. Significance was considered when $\mathrm{P}$ value was $<0.05$. ${ }^{\dagger}$ Significantly different from control (C) group, ${ }^{\ddagger}$ Significantly different from control groups (C and C+Pio), ${ }^{\S}$ Significantly different from HS group

Table 2. Effect of pioglitazone treatment for 14 weeks in a rat model of high sucrose diet on: Serum Glucose, HOMA IR index, Cholesterol (Chol), Triglyceride (TG), Low Density Lipoprotein (LDL), High Density Lipoprotein (HDL)

\begin{tabular}{|c|c|c|c|c|}
\hline & $\mathrm{C}$ & C+Pio & HS & HS+Pio \\
\hline Serum Glu $\left(\mathrm{mg} \mathrm{dL}^{-1}\right)$ & $71.3 \pm 6.3$ & $78.5 \pm 6.4$ & $136.2 \pm 12.3^{\dagger}$ & $95.1 \pm 8.6^{17}$ \\
\hline HOMA index & $0.821 \pm 0.015$ & $0.819 \pm 0.028$ & $3.314 \pm 0.027^{\dagger}$ & $1.053 \pm 0.014^{\dagger+}$ \\
\hline Serum Chol $\left(\mathrm{mg} \mathrm{dL}^{-1}\right)$ & $84.6 \pm 7.5$ & $88.2 \pm 8.4$ & $132.5 \pm 13.4$ & $93.8 \pm 7.9^{\ddagger}$ \\
\hline Serum TG $\left(\mathrm{mg} \mathrm{dL}^{-1}\right)$ & $91.3 \pm 8.2$ & $86.5 \pm 9.1$ & $129.6 \pm 12.8^{\dagger}$ & $106.4 \pm 9.5^{\dagger *}$ \\
\hline Serum LDL $\left(\mathrm{mg} \mathrm{dL}^{-1}\right)$ & $24.7 \pm 3.4$ & $26.7 \pm 3.1$ & $64.8 \pm 6.5^{\dagger}$ & $36.80 \pm 3.4^{\dagger+}$ \\
\hline Serum HDL $\left(\mathrm{mg} \mathrm{dL}^{-1}\right)$ & $46.8 \pm 5.1$ & $44.3 \pm 3.9$ & $28.5 \pm 2.7^{\dagger}$ & $35.2 \pm 2.6^{\dagger \neq}$ \\
\hline
\end{tabular}

C: Control group; C+Pio: Control Pio treated group; HS: High sucrose diet group; HS+Pio: High sucrose diet pioglitazone treated group. Results are expressed as means \pm SD $(n=10)$. Significance was considered when $\mathrm{P}$ value was $<0.05$. 'Significantly different

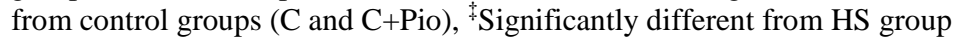

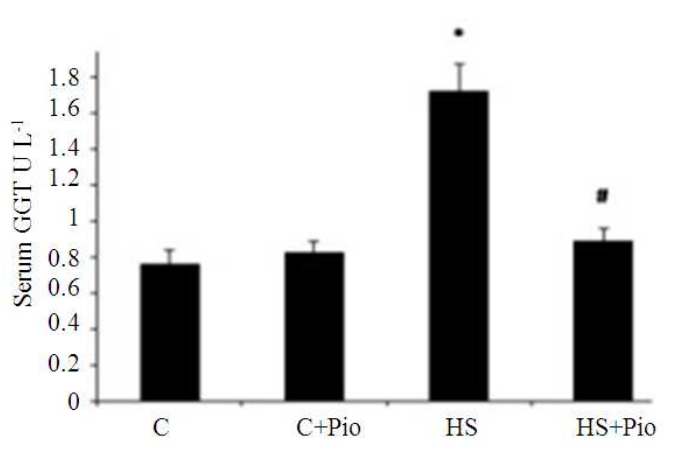

(a)

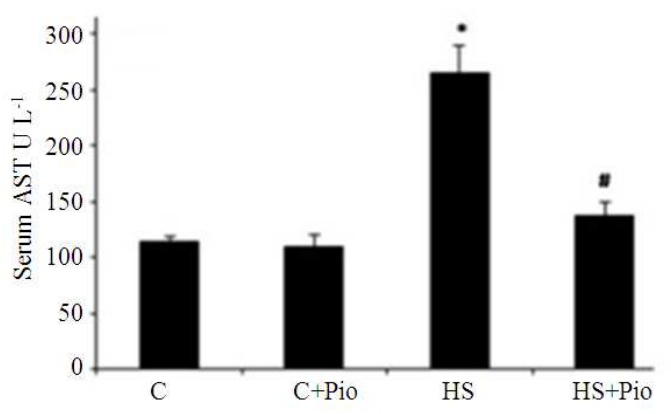

(c)

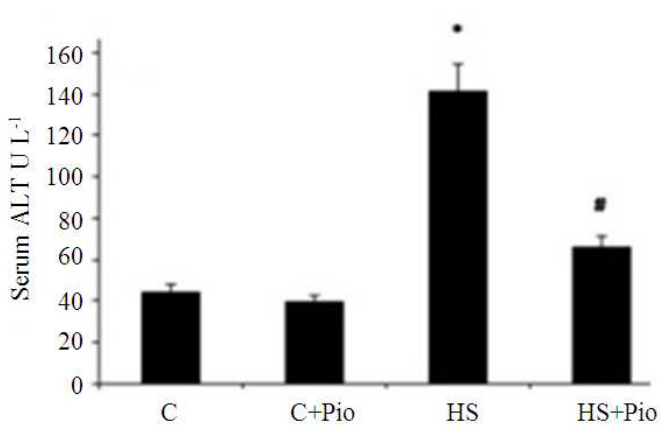

(b)

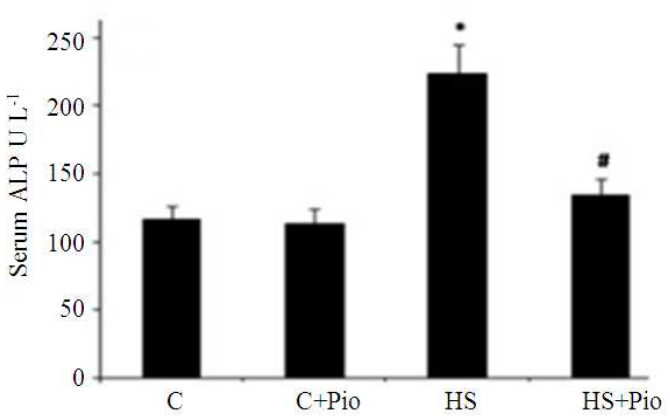

(d)

Fig. 1. Effect of pioglitazone treatment for 14 weeks in a rat model of high sucrose diet on: (a) Serum GGT, (b) Serum ALT, (c) Serum AST and (d) Serum ALP levels. C: Control group; C+Pio: Control Pio treated group; HS: high sucrose diet group; HS+ Pio: High sucrose diet pioglitazone treated group. Results are expressed as means \pm SD $(n=10)$. Significance was considered when $P$ value was $<0.05$. *Significantly different from control groups (C and C+Pio). \# Significantly different from HS group 


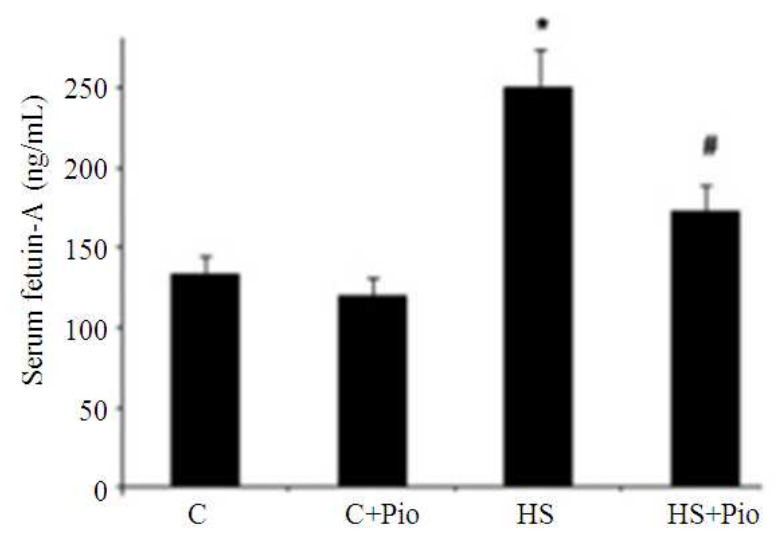

(a)

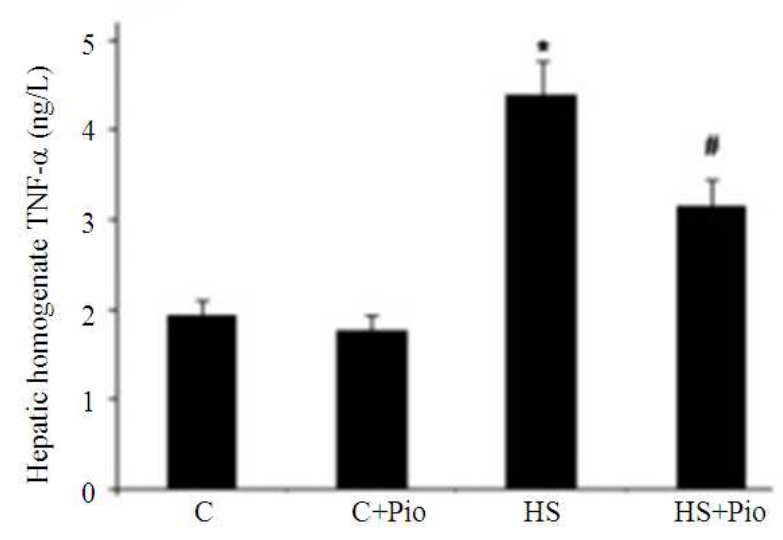

(b)

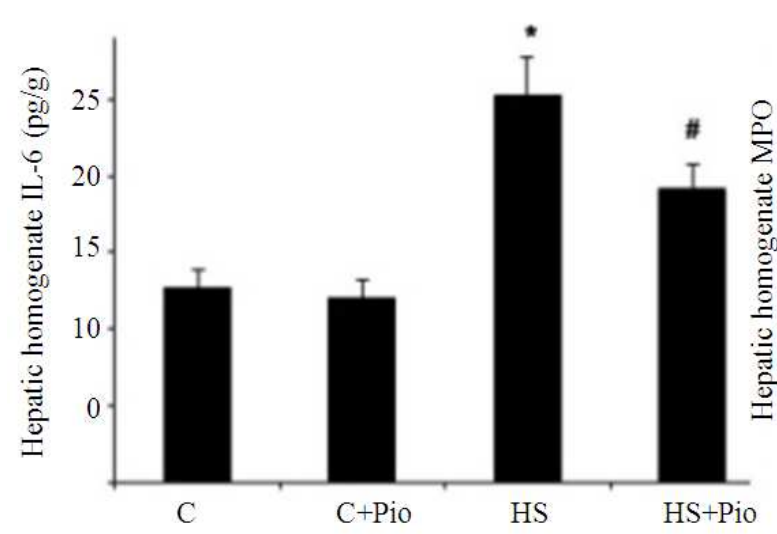

(c)

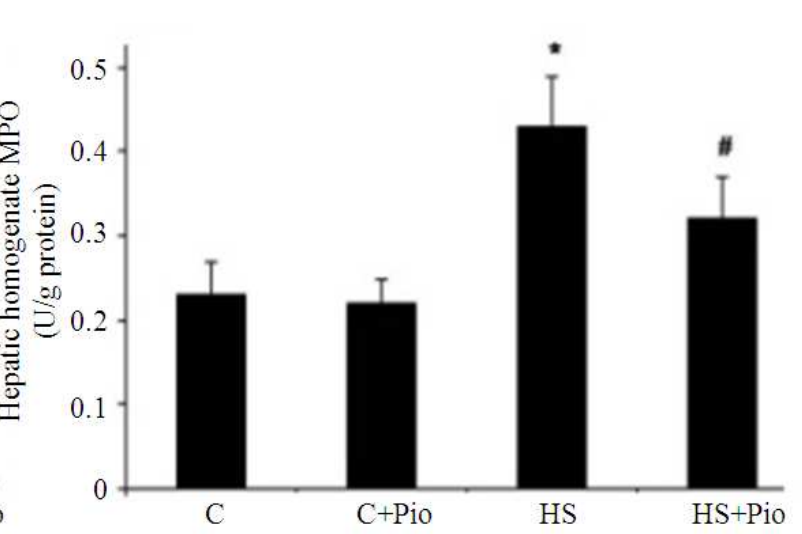

(d)

Fig. 2. Effect of pioglitazone treatment for 14 weeks in a rat model of high sucrose diet on: (a) Serum fetuin-A, (b) hepatic homogenate TNF- $\alpha$, (c) hepatic homogenate IL-6 levels and (d) hepatic homogenate MPO activity. C: Control group; C+Pio: Control Pio treated group; HS: high sucrose diet group; HS+ Pio: High sucrose diet pioglitazone treated group. Results are expressed as means $\pm \mathrm{SD}(\mathrm{n}=10)$. Significance was considered when $\mathrm{P}$ value was $<0.05$. *Significantly different from control groups ( $\mathrm{C}$ and $\mathrm{C}+\mathrm{Pio}$ ). \# Significantly different from HS group.

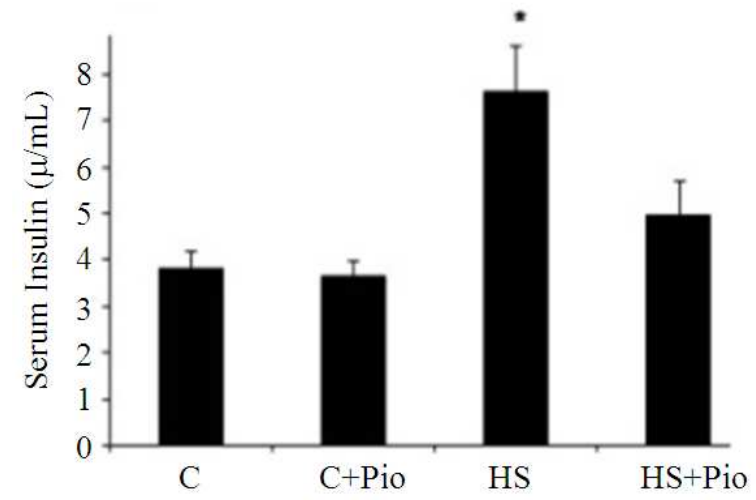

(a)

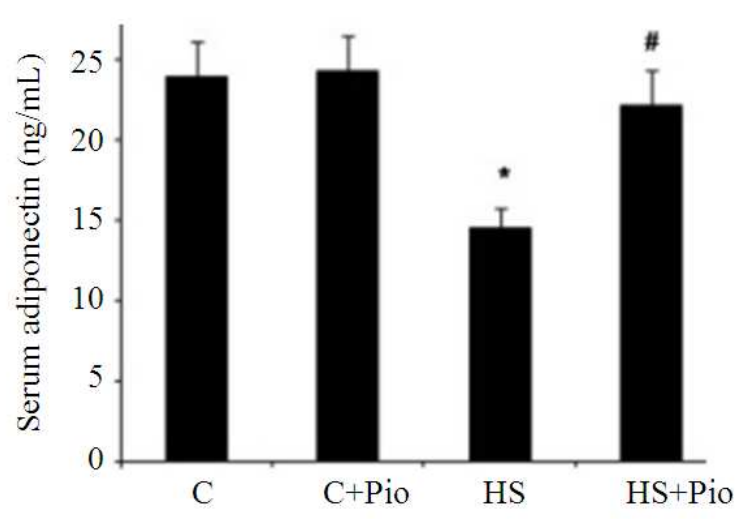

(b) 


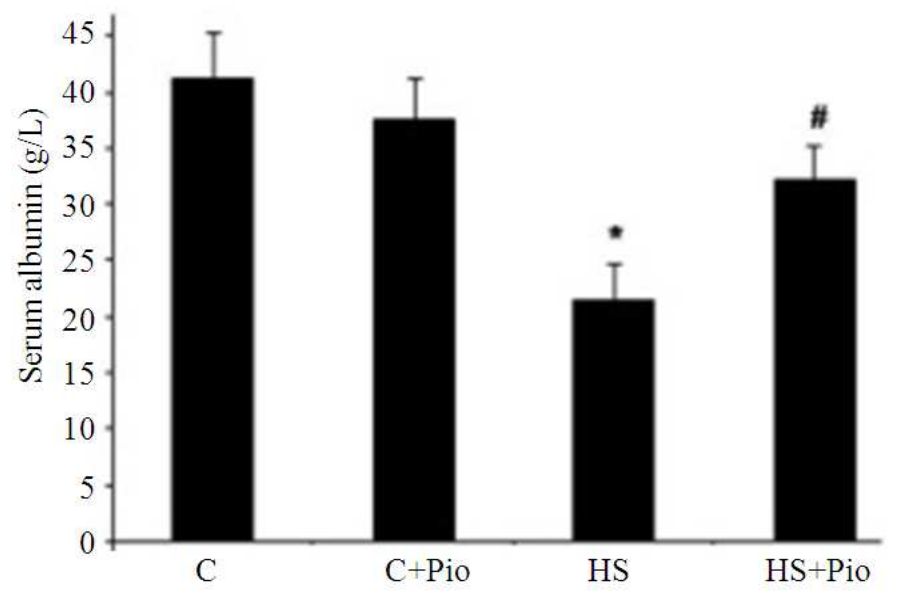

Fig. 3. Effect of pioglitazone treatment for 14 weeks in a rat model of high sucrose diet on: (a) Serum Insulin, (b) Serum Adiponectin and (c) Serum Albumin levels. C: Control group; C+Pio: Control Pio treated group; HS: High sucrose diet group; HS+ Pio: High sucrose diet pioglitazone treated group. Results are expressed as means \pm SD $(n=10)$. Significance was considered when $P$ value was $<0.05$. *Significantly different from control groups (C and C+Pio). \# Significantly different from HS group

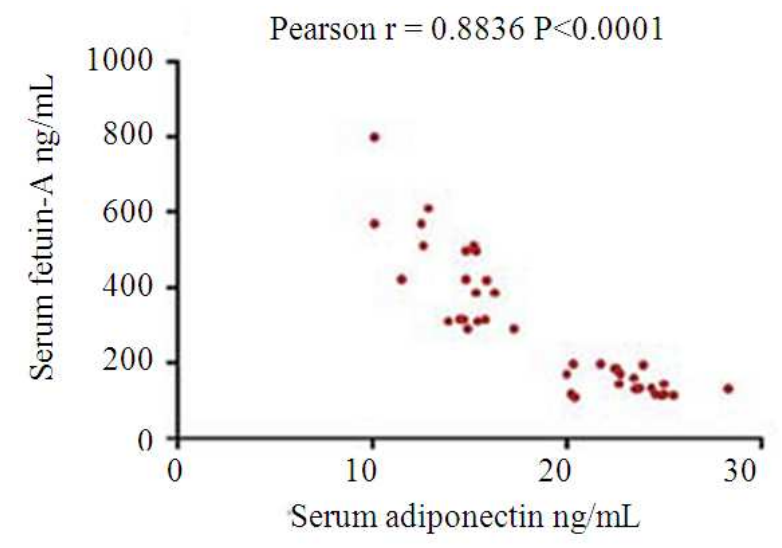

(a)

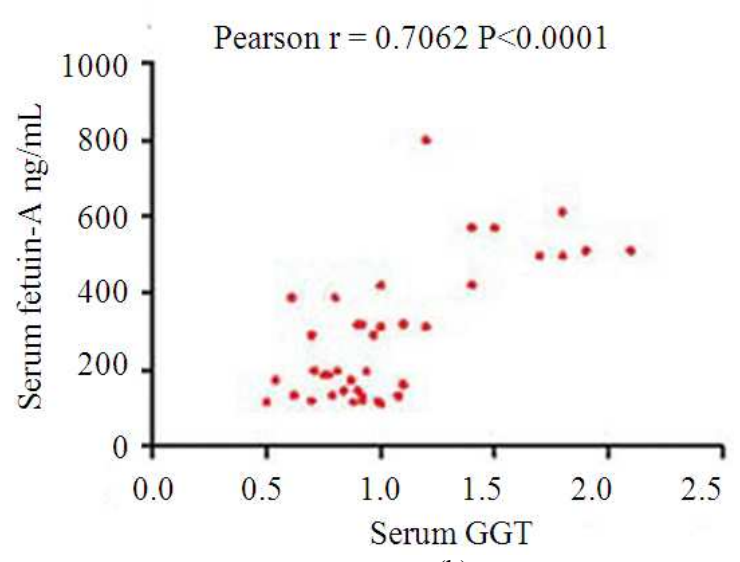

(b)

Fig. 4. Pearson correlation coefficient between serum fetuin-A level with: (a) Serum adiponectin and (b) serum GGT levels. Pearson correlation coefficient between serum fetuin-A level and: (a) Serum adiponectin level for all $(\mathrm{n}=40)(\mathrm{r}=-0.8836)$ $(\mathrm{p}<0.0001)$ and $(\mathrm{b})$ serum level of GGT U L ${ }^{-1}$ for all $(\mathrm{n}=40)(\mathrm{r}=0.7062)(\mathrm{p}<0.0001)$

\subsection{Histopathological Studying of Liver}

The light microscopic examination revealed that a high sucrose diet in HS group resulted in inflammatory cellular infiltration of liver tissue with dilatation of the central vein and discontinuity of the endothelial lining. The blood sinusoids dilated and filled with excessive bleeding and fat deposition. There was an infiltration of dark inflammatory cells with shrunken and pyknotic nuclei (Fig. 5c). Treatment with pioglitazone in HS+Pio resulted in a partial preservation of the hepatic cellular structures with less fat infiltration, while the other hepatocytes appeared with normal morphology and intact nuclei. The hepatic sinusoids showed less bleeding with discontinuity of some its epithelial lining compared with HS group (Fig. 5d). The electron microscopic examination showed that a high sucrose diet in rats resulted in swollen-degenerated hepatocytes with cell membrane discontinuity and damage blood sinusoid and excessive bleeding, disrupted endoplasmic reticulum, abnormal shaped mitochondria, excessive lipid droplets and collagen fibers with swollen nuclei (Fig. 6c). 


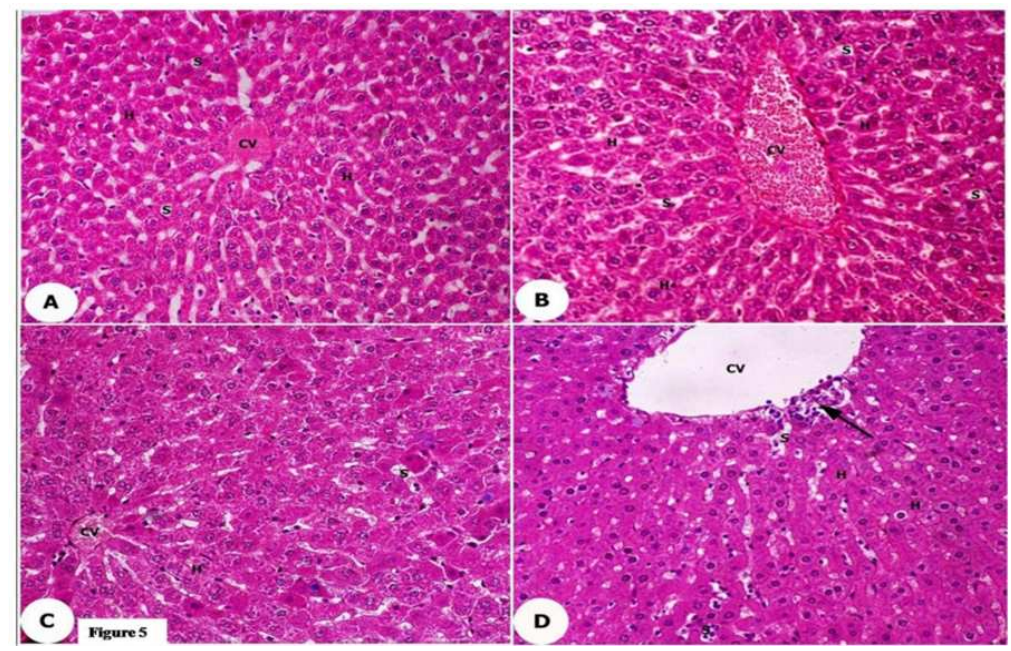

Fig. 5. Light microscopical photomicrograph of: (a) control liver tissues, (b) control Pioglitazone liver tissues, (c) high sucrose diet group, (d) high sucrose diet treated with Pioglitazone groups. Light micrograph of showing: (a) Control liver tissues with preserved normal polygonal shape hepatocytes, round central nuclei with normal hepatic sinusoids; (b) Liver tissue of control rats treated with pioglitazone normal hepatocytes with normal cellular architecture; (c) Liver tissue of high sucrose diet rat infiltrated by dark inflammatory cells with shrunken pyknotic nuclei, dilated central vein with discontinuity of the endothelial cells and blood sinusoids filled with excessive bleeding; (d) Liver tissue of high sucrose diet rat treated with pioglitazone having scanty cellular fat vacuolation. The hepatic sinusoids showed less bleeding with discontinuity of some its epithelial lining while the majority of hepatocytes appear with normal morphology and intact nuclei compared with HS group (X 400)

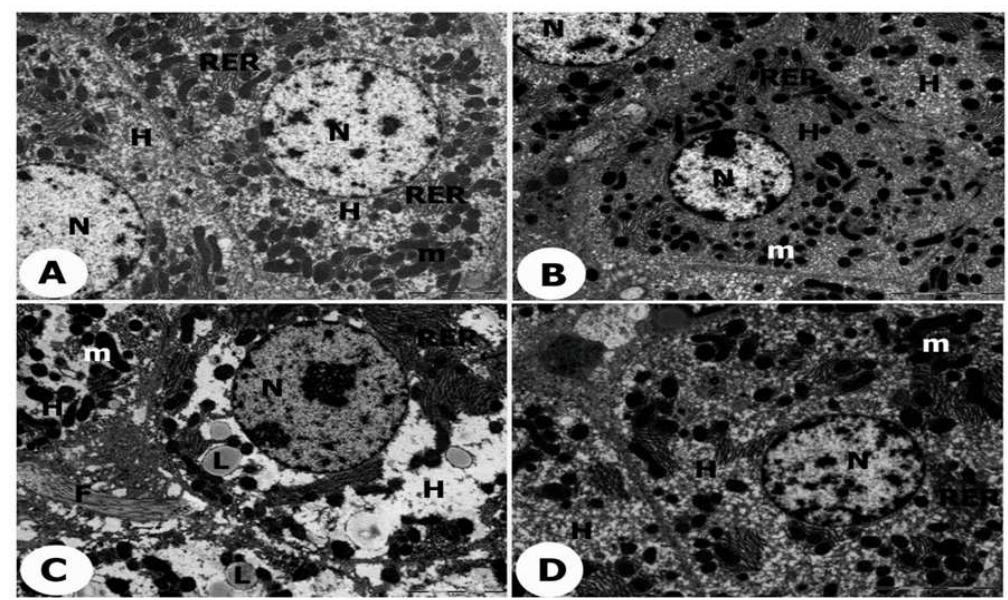

Fig. 6. Electron microscopic transmission photomicrograph of: (a) Control liver tissues, (b) control Pioglitazone liver tissues, (c) high sucrose Diet group, (d) high sucrose diet treated with Pioglitazone groups. Electron micrograph of: [a] Liver tissue of control rat $(\mathrm{C})$ shows normal Hepatocytes $(\mathrm{H})$ with normal nucleus $(\mathrm{N})$, rough endoplasmic reticulum (RER) and mitochondria $(\mathrm{m})$. White arrow indicates intact cell membrane. [b] Liver tissue of control rat treated with Pio (C+Pio) shows normal hepatocytes having intact normal nucleus and other cellular organelles. [c] Liver tissue of high sucrose diet rat showing swollen degenerating Hepatocytes $(\mathrm{H})$ with cell membrane discontinuity and damaged blood sinusoid with excess bleeding (S). There were disruption of the endoplasmic reticulum, abnormal shaped mitochondria, excessive Lipid droplets (L), collagen Fibers (F) and swollen nucleus. [d] High sucrose diet with pioglitazone treatment rat (HS+Pio) shows improving the hepatocellular architures $(\mathrm{H})$ with intact cell membrane and less fat vacuolation and collagen fibers. There was normal histological appearance of both endoplasmic reticulum and mitochondria with intact nucleus. Endothelial cells of the blood sinusoids contain some Vacuoles (V) with intact nucleus. (X 5000) 
Whereas Pio treatment in HS+Pio rats improved the hepatocytes and the cell membrane structure with less fat vacuolation and collagen fibers and normal histological appearance of both endoplasmic reticulum and mitochondria with a healthy nucleus (Fig. 6d).

\section{DISCUSSION}

Several studies documented that diet composition is indicted in the development of obesity and insulin resistance (Bessesen, 2001). Fourteen weeks of high sucrose diet in rats resulted in a significant increase in body weight accompanied with hyperglycemia, dyslipidemia and hyper-insulinemia with insulin resistance development. This induced hepatic inflammatory reaction revealed by a significant elevation of the hepatic inflammation markers TNF- $\alpha$, IL- 6 , fetuinA levels and MPO activity reflecting the number of polymorphonuclear neutrophils in the liver. Hepatic inflammation deteriorated the liver function proved by an elevation of serum GGT, ALT, AST and ALP levels together with a reduction of serum albumin significantly compare with control groups. The light microscopic study showed hepatocellular infiltration with inflammatory cells and excessive fat vacuolation with pyknotic nuclei. The central veins dilated and the endothelial lining of the hepatic sinusoids showed discontinuity with excessive bleeding. All these finding confirmed the biochemical and metabolic disturbances induced by a high sucrose diet in rats. The electron microscopic examination of the liver tissue of the HS rats revealed degeneration of the hepatocytes with an abnormal cell membrane, excessive vacuolar degeneration and collagen fibers with swollen nucleus and damage blood sinusoid. The cellular organelles showed disrupted endoplasmic reticulum and swollen mitochondria with cristae destruction. Our results are in agreement with several studies (Yoshimoto et al., 1997; Yang et al., 2005). Few researches concerned with the hepatic anti-inflammatory effect of PPAR- $\gamma$ agonist pioglitazone at the level of ultra-structure changes and its relation to improvement of insulin sensitivity, fetuin-A and adiponectin levels in high sucrose diet. The aim of this study was to investigate the hepatic antiinflammatory effect of the insulin sensitizer pioglitazone on the hepatic, ultra-structure and metabolic changes induced by a high sucrose diet in rats. In addition, we addressed the mechanisms by which pioglitazone mediates its anti-inflammatory action.

Pioglitazone is a specific potent PPAR- $\gamma$ ligand having substantial anti-inflammatory accompanied its insulin sensitizing activities. Moller and Berger (2003) documented the presence of a higher level of PPAR- $\gamma$ expression in the liver of obese rats and its activation in addition to the insulin sensitizing effect has selected antiinflammatory activity. The mechanisms of the antiinflammatory and insulin-sensitizing activities of PPAR$\gamma$ ligands are complex. It has been reported that PPAR- $\gamma$ activation by pioglitazone induces expression of fatty acid transporters involved in fatty acid catabolism such as lipoprotein lipase, fatty acid acetyl-CoA synthase, oxidase and carnitine palmitoyl-transferase I (Berger and Moller, 2002). Activation of PPAR- $\gamma$ in adipocytes induces the expression of many PPRE-containing genes involved in lipid metabolism. This could lead to a gradual increase in liver insulin sensitivity (Gurnell, 2006). Improvement of lipid metabolism by PPAR- $\gamma$ reduced the circulating level of FFAs and a more gradual reduction in liver steatosis (Guan et al., 2002). This proved in the present study by the significant improvement of the dyslipidemia, the elevated liver enzymes and the serum albumin in the Pio treated group.

An important contributor to the insulin-sensitizing efficacy of PPAR- $\gamma$ agonists is the suppression of local and systemic cytokine production (Moller and Berger 2003). It has been found that treatment of the obese patients with PPAR- $\gamma$ agonists reduces circulating levels of inflammatory cytokines and other proinflammatory markers leading to improve insulin sensitivity. Samaha et al. (2006) reported that binding of PPAR- $\gamma$ to co-activators reduces the levels of coactivators available for binding to pro-inflammatory transcription factors such as NF- $\mathrm{BB}$. This causes a decrease in transcription of a number of pro inflammatory genes including various interleukins and tumor necrosis factors. This was confirmed in the present study by the significant reduction in hepatic inflammatory markers TNF- $\alpha$, IL-6, fetuin-A levels and MPO activity in the Pio treated group (HS+Pio group). Importantly, it has been reported that, PPAR- $\gamma$ agonists can potently suppress TNF- $\alpha$ production and its action in adipocytes. This could result in ablation of TNFalpha's ability to inhibit insulin signaling and to down regulate the glucose transporter expression leads to inhibition of TNF- $\alpha$ induced insulin resistance and other hepatic adverse effects (Moller, 2000). The light microscopic histopathological examination revealed an improvement of hepatocytes, structure and their cell membrane with less dilation of the central vein. The hepatic sinusoids showed less bleeding with regular endothelial lining. While electron microscopic transmission study in the Pio treated group showed continuity of the hepatic cell membrane and preseved 
cellular structure with less fat vacuolation and collagen fibers. The hepatocellular organelles revealed a normal histological appearance of the endoplasmic reticulum and the mitochondria with regular cristae and a normal nuclear histological appearance.

Recent research has shown that PPAR- $\gamma$ agonists increase plasma level of adiponectin in obese animal models and type II diabetic patients (Kadowaki et al., 2006). Our study showed a significant increase in the adiponectin level in high sucrose diet rats treated with Pio. Induction of adiponectin secretion by PPAR- $\gamma$ agonists might have a key role in the mechanism by which Pio treatment ameliorates the hepatic inflammatory processes induced by high sucrose diet. Recent evidences suggested that the normal level of adiponectin inhibits the progression of hepatic steatosis to fibrosis through an AMPK-dependent pathway (Ix and Sharma, 2010).

Different other mechanisms of the anti-inflammatory activity of Pio treatment have been reported. Pioglitazone has the ability to attenuate the diet induced the expression of the inflammatory enzymes iNOS and COX-2 whose role in the development of insulin resistance has been recently documented in obese rats and in cultured hepatocytes (Fujimoto et al., 2005; Hsieh et al., 2009). Other studies suggested that PPAR$\gamma$ activation suppresses the monocyte-macrophage migration, the expression of the adhesion molecule ICAM-1 (Moore et al., 2001), the inflammatory action of eicosanoid leukotriene B4 (Devchand et al., 1996), the induction of apoptosis in cytokine-activated macrophages and NF-kB signaling (Delerive et al., 2001). It has been reported that PPAR- $\gamma$ agonists reduce adipose 11b-HSD-1 level, "the enzyme regulates the conversion of cortisone to cortisol". Since hypercorticosteroidism is a well-known cause of insulin resistance with subsequent inflammatory cascades in obese patients (Masuzaki et al., 2001).The limitation of our study was we didn't investigate the hepatic enzymes homogenate levels, pancreatic tissue ultra-structure changes and insulin gene expression that could highlighting on the hepato-protective mechanism of Pio.

\section{CONCLUSION}

The present study suggests that pioglitazone as a member of PPAR- $\gamma$ agonist insulin sensitizer has a hepatic anti-inflammatory activities through different mechanisms. It has the ability to reduce the serum lipids, hepatic fat accumulation, cytokines overproduction and insulin resistance resulting in amelioration of hepatic inflammatory reaction induced by high sucrose diet. Adiponectin may play a major hepatic anti-inflammatory role in such condition treated with Pio. The improvement by the pioglitazone treatment was not only at the level of biochemical changes, but also at the histopathological and ultrastructures changes. Further studies should be carried out on hepato-protective role of Pio treatment in order to uncover the exact mechanism of action. Also, investigation of Pio with different doses and therapy regimens in other animal tissues for ultra-structure changes is required to ensure its efficacy and safety.

\section{ACKNOWLEGEMENT}

We kindly appreciate all staff members and technicians of the Physiology and Biochemistry Labs, College of Medicine, King Khalid University, Saudi Arabia and College of Medicine, Menoufiya University, Egypt; for their great help in every step in this study. Special thanks to Dr. Mohamed Soliman for his kind help in the biochemical investigation and to Mrs. Asmaa E Hassan for her great effort during the preparation of the statistics for this manuscript.

\section{CONFILICT INTEREST}

The researchers declare that they have no competing interests.

\section{REFERENCES}

Berger, J. and D.E. Moller, 2002. The mechanism of action of PPARs. Ann. Rev. Med., 53: 409-435. DOI: 10.1146/annurev.med.53.082901.104018

Bessesen, D.H., 2001. The role of carbohydrates in insulin resistance. J. Nut., 131: 2782S-2786S. PMID: 11584106

Collino, M., M. Aragno, S. Castiglia, G. Miglio and C. Tomasinelli et al., 2010. Pioglitazone improves lipid and insulin levels in overweight rats on a high cholesterol and fructose diet by decreasing hepatic inflammation. Br. J. Pharmacol., 160: 1892-1902. DOI: $10.1111 /$ j.1476-5381.2010.00671.x

Delerive, P., J.C. Fruchart and B. Staels, 2001. Peroxisome proliferator activated receptors in inflammation control. J. Endocrinol., 169: 453-459. DOI: $10.1677 /$ joe.0.1690453

Devchand, P.R., H. Keller, J.M. Peters, M. Vazquez and F.J. Gonzalez et al., 1996. The PPAR alphaleukotriene B4 pathway to inflammation control. Nature, 384: 39-43. DOI: 10.1038/384039a0 
Fujimoto, M., N. Shimizu, K. Kunii, J.A. Martyn and K. Ueki et al., 2005. A role for iNOS in fasting hyperglycemia and impaired insulin signaling in the liver of obese diabetic mice. Diabetes, 54: 13401348. DOI: $10.2337 /$ diabetes.54.5.1340

Gerstein, H.C., S. Yusuf, J. Bosch, J. Pogue and P. Sheridan et al., 2006. Effect of rosiglitazone on the frequency of diabetes in patients with impaired glucose tolerance or impaired fasting glucose: A randomized controlled trial. Lancet, 368: 10961105. DOI: 10.1016/S0140-6736(06)69420-8

Guan, H.P., Y. Li, M. Jensen, C. Newgard and C. Steppan et al., 2002. Futile metabolic cycle activated in adipocytes by antidiabetic agents. Nat. Med., 8: 1122-1128. DOI: 10.1038/nm780

Gunduz, F.O., S.T. Yildirmak, M. Temizel, Y. Faki and M. Cakmak et al., 2011. Serum visfatinan fetuin-a levels and glycemic control in patients with obese type 2 diabetes mellitus. Diabetes Metab. J., 35: 523-528. DOI: 10.4093/dmj.2011.35.5.523

Gurnell, M., 2006. Peroxisome proliferator-activated receptor gamma and the regulation of adipocyte function: Lessons from human genetic studies. Best Pract. Res. Clin. Endocrinol. Metab., 19: 501-23. DOI: $10.1016 /$ j.beem.2005.10.001

Al-Musa, H. and F. AL-Hashem 2014. Hypoglycemic, hepato-renal and antioxidant potential effects of chamomile recutita flowers ethanolic extract in streptozotocin-diabetic rats. Am. J. Pharmacol. Toxicol., 9: 1-12. DOI: 10.3844/ajptsp.2014.1.12

Hsieh, P.S., J.S. Jin, C.F. Chiang, P.C. Chan and C.H. Chen et al., 2009. COX-2-mediated inflammation in fat is crucial for obesity-linked insulin resistance and fatty liver. Obesity, 17: 1150-1157. PMID: 19247274

Ix, J.H. and K. Sharma, 2010. Mechanisms linking obesity, chronic kidney disease and fatty liver disease: The roles of fetuin-a, adiponectin and AMPK. J. Am. Soc. Nephrol., 21: 406-412. DOI: 10.1681/ASN.2009080820

Kadowaki, T., T. Yamauchi and N. Kubota, 2008. The physiological and pathophysiological role of adiponectin and adiponectin receptors in the peripheral tissues and CNS. FEBS Lett., 582: 74-80. DOI: 10.1016/j.febslet.2007.11.070

Kadowaki, T., T. Yamauchi, N. Kubota, K. Hara and K. Ueki et al., 2006. Adiponectin and adiponectin receptors in insulin resistance, diabetes and the metabolic syndrome. J. Clin. Invest., 116: 17841792. DOI: $10.1172 / \mathrm{JCI} 29126$
Ismail, T.A., M.M. Soliman and S.A. Ismail, 2014. Adiponectin regulation in type 2 diabetic Rats: Effects of insulin, metformin and dexamethasone. Am. J. Pharmacol. Toxicol., 8: 197-208. 2013 DOI: 10.3844/ajptsp.2013.197.208

Koufany, M., D. Moulin, A. Bianchi, M. Muresan and S. Sebillaud et al., 2008. Anti-inflammatory effect of antidiabetic thiazolidinediones prevents bone resorption rather than cartilage changes in experimental polyarthritis. Arthritis Res. Ther., 10: R6-R6. DOI: 10.1186/ar2354

Kubota, N., Y. Terauchi, T. Yamauchi, T. Kubota and M. Moroi et al., 2002. Disruption of adiponectin causes insulin resistance and neointimal formation. J. Biol. Chem., 277: 25863-25866. DOI: 10.1074/jbc.C200251200

Lee, M.H., R.L. Klein, H.M. El-Shewy, D.K. Luttrell and L.M., Luttrell, 2008. The adiponectin receptors AdipoR1 and AdipoR2 activate ERK1/2 through a Src/Ras-dependent pathway and stimulate cell growth. Biochemistry, 47: 11682-11692. DOI: 10.1021/bi801451f

Lombardo, Y.B., S. Drago, A. Chicco, P. Fainstein-Day and R. Gutman et al., 1996. Long-term administration of a sucrose-rich diet to normal rats: Relationship between metabolic and hormonal profiles and morphological changes in the endocrine pancreas. Metabolism, 45: 1527-1532. DOI: 10.1016/S0026-0495(96)90183-3

Masuzaki, H., J. Paterson, H. Shinyama, N. Morton and J. Mullins et al., 2001. A transgenic model of visceral obesity and the metabolic syndrome. Science, 294: 2166-2170. DOI: 10.1126/science. 1066285

Matthews, D.R., J.P. Hosker, A.S. Rudenski, B.A. Naylor and D.F. Treacher et al., 1985. Homeostasis model assessment: Insulin resistance and b-cell function from fasting plasma glucose and insulin concentrations in man. Diabetologia, 28: 412-419. DOI: $10.1007 / \mathrm{BF} 00280883$

Michalik, L., J. Auwerx, J.P. Berger, V.K. Chatterjee and C.K. Glass et al., 2006. International union of pharmacology. LXI. Peroxisome proliferatoractivated receptors. Pharmacol. Rev., 58: 726-41. DOI: $10.1124 /$ pr.58.4.5

Moller, D., 2000. Potential role of TNF- $\alpha$ in the pathogenesis of insulin resistance and type 2 diabetes. Trends. Endocrinol. Metab., 11: 212-217. DOI: 10.1016/S1043-2760(00)00272-1

Moller, D.E. and J.P. Berger, 2003. Role of PPARs in the regulation of obesity related insulin sensitivity and inflammation. Int. J. Obes. Relat. Metab. Disord., 27: S17-S21. DOI: 10.1038/sj.ijo.0802494 
Moore, K., M. Fitzgerald and M. Freeman, 2001. Peroxisome proliferator activated receptors in macrophage biology: Friend or foe? Curr. Opin. Lipido., 12: 519-527. DOI: 10.1097/00041433200110000-00007

Muñoz Cano, J.M., A.C. Aguilar and J.C. Hernández, 2013. Lipid-lowering effect of maize-based traditional Mexican food on a metabolic syndrome model in rats. Lipids Health Dis., 15: 12-35. DOI: 10.1186/1476-511X-12-35

Nielsen, S.J. and B.M. Popkin, 2004. Changes in beverage intake between 1977 and 2001. Am. J. Prev. Med., 27: 205-210. DOI: 10.1016/j.amepre.2004.05.005

Bashir, S.O., M.D. Morsy, H.F. Sakr, H.M. El Refaey and R.A. Eid et al., 2014. Quercetin ameliorates diabetic nephropathy in rats via modulation of renal $\mathrm{Na}+, \mathrm{K}+$-atpase expression and oxidative stress. Am. J. Pharmacol. Toxicol., 9: 84-95. DOI: 10.3844/ajptsp.2014.84.95

Rauth, G., O. Poschke, E. Fink, M. Eulitz and S. Tippmer et al., 1992. The nucleotide and partial amino acid sequences of rat fetuin. Identity with the natural tyrosine kinase inhibitor of the rat insulin receptor. Eur. J. Biochem., 204: 523-529. DOI: 10.1111/j.1432-1033.1992.tb16663.x
Samaha, F.F., P.O. Szapary, N. Iqbal, M.M. Williams and L.T. Bloedon et al., 2006. Effects of rosiglitazone on lipids, adipokines and inflammatory markers in nondiabetic patients with low highdensity lipoprotein cholesterol and metabolic syndrome. Arterioscler. Thromb. Vasc. Biol., 26: 624-630. DOI: 10.1161/01.ATV.0000200136.56716.30

Xu, S.Q., Y.H. Li, S.H. Hu, K. Chen and L.Y. Dong, 2008. Effects of Wy14643 on hepatic ischemia reperfusion injury in rats. World J. Gastroenterol., 14: 6936-6942. DOI: 10.3748/wjg.14.6936

Yang, B., P. Lin, K.M. Carrick, J.A. McNulty and L.G. Clifton et al., 2005. PPAR gamma agonists diminish serum VEGF elevation in diet-induced insulin resistant SD rats and ZDF rats. Biochem. Biophys. Res. Commun., 334: 176-182. DOI: 10.1016/j.bbrc.2005.06.078

Yoshimoto, T., M. Naruse, M. Nishikawa, K. Naruse and A. Tanabe et al., 1997. Antihypertensive and vasculo- and renoprotective effects of pioglitazone in genetically obese diabetic rats. Am. J. Physiol., 272: E989-E996. PMID: 9227442 